\title{
The Projective Line over the Integers
}

\author{
Ela Celikbas and Christina Eubanks-Turner
}

\begin{abstract}
In this article we survey work that has been done to describe the partially ordered set of prime ideals of the projective line over the integers, denoted by $\operatorname{Proj}(\mathbb{Z}[h, k])$. We consider a conjecture given by Aihua Li and Sylvia Wiegand that would complete a characterization of $\operatorname{Proj}(\mathbb{Z}[h, k])=\operatorname{Spec}(\mathbb{Z}[x]) \cup \operatorname{Spec}\left(\mathbb{Z}\left[\frac{1}{x}\right]\right)$. In this paper we give some previous results and some new results that support the conjecture. In particular we show that a possible axiom for $\operatorname{Proj}(\mathbb{Z}[h, k])$ proposed by Arnavut, Li and Wiegand holds for a large number of previously unknown cases.
\end{abstract}

Keywords. Prime Ideals, Prime Spectrum, Projective Line, Polynomials, Radical Element.

2010 Mathematics Subject Classification. 13A15, 13B25, 06A11, 06A06.

\section{Introduction}

Let $h$ and $k$ be indeterminates over the integers $\mathbb{Z}$. The projective line $\operatorname{Proj}(\mathbb{Z}[h, k])$ over the integers can be viewed as the partially ordered set under inclusion of all prime ideals of $\mathbb{Z}[h, k]$ that are generated by finite sets of homogeneous polynomials in $h$ and $k$ other than those prime ideals that contain both $h$ and $k$. For $x$ an indeterminate over $\mathbb{Z}$, the prime spectrum of $\mathbb{Z}[x]$ or $\operatorname{Spec}(\mathbb{Z}[x])$, the partially ordered set of prime ideals of $\mathbb{Z}[x]$ under inclusion, is sometimes called the affine line over $\mathbb{Z}$. In this paper we let $x=h / k$ and we view $\operatorname{Proj}(\mathbb{Z}[h, k])$ as the union of its affine pieces $\operatorname{Spec}(\mathbb{Z}[x])$ and $\operatorname{Spec}\left(\mathbb{Z}\left[\frac{1}{x}\right]\right)$. In this view of $\operatorname{Proj}(\mathbb{Z}[h, k])$, the intersection of $\operatorname{Spec}(\mathbb{Z}[x])$ with $\operatorname{Spec}\left(\mathbb{Z}\left[\frac{1}{x}\right]\right)$ is identified with $\operatorname{Spec}\left(\mathbb{Z}\left[x, \frac{1}{x}\right]\right)$; cf. Notation 2.7 (ii).

In 1986, Roger Wiegand gave five axioms that characterized the prime spectrum of $\mathbb{Z}[x]$ as a partially ordered set; cf. [7] and Definition 2.4 below. Four of those axioms hold for $\operatorname{Proj}(\mathbb{Z}[h, k])$, but $\operatorname{Proj}(\mathbb{Z}[h, k])$ fails to satisfy the key fifth axiom of $\operatorname{Spec}(\mathbb{Z}[x])$; cf. [5]. So far no one has completed a characterization of $\operatorname{Proj}(\mathbb{Z}[h, k])$, although there have been several related results. In 1994 William Heinzer, David Lantz and Sylvia Wiegand determined those partially ordered sets that occur as the projective line $\operatorname{Proj}(R[h, k])$ when $R$ is a one-dimensional semilocal domain. In 1997, Aihua $\mathrm{Li}$ and Sylvia Wiegand described some properties of $\operatorname{Proj}(\mathbb{Z}[h, k])$. In 2002, Meral Arnavut conjectured that a modified form of the key axiom of $\operatorname{Spec}(\mathbb{Z}[x])$ would complete a characterization of $\operatorname{Proj}(\mathbb{Z}[h, k])$; she gave partial results toward her conjecture; cf. [1] and Axiom 4.2 below.

The key axiom for $\operatorname{Spec}(\mathbb{Z}[x])$ stipulates the existence of "radical elements", defined in Definition 2.2, for pairs $(S, T)$ of finite subsets of $\operatorname{Spec}(\mathbb{Z}[x])$, where the elements 
of $S$ have height one and those of $T$ have height two. Radical elements often exist for sets $S$ and $T$ in $\operatorname{Proj}(\mathbb{Z}[h, k])$, but not always. We expect that the determination of when radical elements exist would lead to a characterization of $\operatorname{Proj}(\mathbb{Z}[h, k])$.

In this article we continue the investigation of the projective line over the integers. In the process we give further evidence for Arnavut's conjecture. Among our main results are new cases when radical elements exist, such as Theorem 5.5 and Theorem 5.8. In Theorem 5.5, we show the existence of radical elements when every maximal ideal of $T \cap \operatorname{Spec}(\mathbb{Z}[x])$ has form $(x, p) \mathbb{Z}[x]$, where $p$ is a prime integer; each $(x, p)$ corresponds to exactly one maximal ideal of form $\left(\frac{1}{x}, p\right) \mathbb{Z}\left[\frac{1}{x}\right] \in T \cap \operatorname{Spec}\left(\mathbb{Z}\left[\frac{1}{x}\right]\right)$; and vice versa. In Theorem 5.8, we find radical elements for sets of form

$$
\begin{aligned}
S & =\left\{\left(p_{1}\right), \ldots,\left(p_{n}\right),(x),\left(\frac{1}{x}\right),(x-a),(x-b)\right\}, \quad \text { and } \\
T & =\left\{\left(x, p_{1}\right), \ldots,\left(x, p_{\ell}\right),\left(\frac{1}{x}, p_{\ell+1}\right), \ldots,\left(\frac{1}{x}, p_{n}\right)\right\},
\end{aligned}
$$

where the $p_{i}$ are prime integers relatively prime to $a, b \in \mathbb{Z}$, under certain conditions. It is difficult to produce prime ideals that are the correct radical elements. For the proof of Theorem 5.5, we use Hilbert's Irreducibility Theorem to find radical elements. For the proof of Theorem 5.8, we use Euler's theorem. Theorem 5.8 is a special case of the conjecture and answers a question in Arnavut's paper [1].

In Section 2 we restate relevant notation, definitions and previous results of Meral Arnavut, Aihua Li and Sylvia Wiegand from [1], [4], and [5]. In Section 3 we discuss the coefficient subset of $\operatorname{Proj}(\mathbb{Z}[h, k])$ from $[4]$. A coefficient subset of $\operatorname{Proj}(\mathbb{Z}[h, k])$ behaves like the set of all prime ideals of $\operatorname{Proj}(\mathbb{Z}[h, k])$ generated by prime integers. In Section 4 we summarize Meral Arnavut's results towards the conjecture. Our new results are in Section 5; they all support the conjecture.

\section{Definitions and Background}

First we establish some notation.

Notation 2.1. Let $\mathbb{N}$ be the natural numbers and let $\mathbb{Q}$ be the rationals. Let $U$ be a partially ordered set, sometimes abbreviated poset. A chain in $U$ is a totally ordered subset of $U$. Throughout we suppose that $U$ has a unique minimal element $u_{0}$ and that every chain in $U$ is finite.

For $u \in U$, the height of $u, \operatorname{ht}(u)$, is the length $t$ of a maximal length chain in $U$ of form $u_{0}<u_{1}<u_{2}<\cdots<u_{t}=u$; the dimension of $U, \operatorname{dim}(U)$, is the maximum of $\{h t(u) \mid u \in U\}$; and we set

$$
H_{1}(U):=\{u \in U \mid \operatorname{ht}(u)=1\}, \quad \text { and } \quad H_{2}(U):=\{u \in U \mid \operatorname{ht}(u)=2\} .
$$


For every $u, v \in U$ and $S \subseteq H_{1}(U)$, define

$$
\begin{gathered}
u^{\uparrow}:=\{v \in U \mid u<v\}, \quad v^{\downarrow}:=\{u \in U \mid u<v\}, \quad(u, v)^{\uparrow}:=u^{\uparrow} \cap v^{\uparrow}, \quad \text { and } \\
S^{\uparrow}=\left\{t \in U \mid t \in s^{\uparrow}, \text { for all } s \in S\right\}
\end{gathered}
$$

For $U:=\operatorname{Spec}(R)$, where $R$ is a Noetherian ring, and $a \in R$, let

$$
\begin{aligned}
a^{\uparrow} & :=\{P \in \operatorname{Spec}(R) \mid a \in P\} \quad \text { and } \\
(a, b)^{\uparrow}: & =\{P \in \operatorname{Spec}(R) \mid a \in P \text { and } b \in P\} .
\end{aligned}
$$

In Definition 2.4, we give the five axioms that Roger Wiegand showed characterize $\operatorname{Spec}(\mathbb{Z}[x])$ as a partially ordered set; cf. [7]. The key axiom is easier to state if we first define "radical element".

Definition 2.2 ([4]). Let $U$ be a partially ordered set of dimension two and let $S$ and $T$ be finite subsets of $U$ such that $\emptyset \neq S \subseteq H_{1}(U)$ and $T \subseteq H_{2}(U)$. If $w \in H_{1}(U)$ satisfies (i) and (ii), then $w$ is called a radical element for $(S, T)$ :

(i) $w<t$, for every $t \in T$,

(ii) Whenever $m \in U$ is greater than both $w$ and $s$, for some $s \in S$, then $m \in T$.

(In other words, $w$ is a radical element for $(S, T)$ if and only if $\bigcup_{s \in S}(w, s)^{\uparrow} \subseteq$ $T \subset w^{\uparrow}$.)

The following picture illustrates the relations between a radical element and the associated sets $S$ and $T$ in a two-dimensional poset:

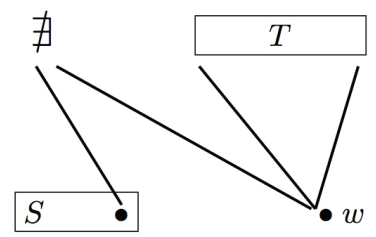

Figure 1. Relations for a radical element $w$.

For convenience we also introduce the following notation that is used later.

Notation 2.3. A ht(1,2)-pair of a poset $U$ is a pair $(S, T)$ of finite subsets $S$ and $T$ of $U$ such that $\varnothing \neq S \subseteq H_{1}(U)$ and $T \subseteq H_{2}(U)$.

Definition 2.4. Let $U$ be a partially ordered set. The following five axioms are called the Countable Integer Polynomial (CZP) Axioms:

(P1) $U$ is countable and has a unique minimal element.

(P2) $U$ has dimension two. 
(P3) For each element $u$ of height-one, $u^{\uparrow}$ is infinite.

(P4) For each pair $u, v$ of distinct elements of height-one, $(u, v)^{\uparrow}$ is finite.

(RW) Every ht(1,2)-pair of $U$ has at least one radical element in $U$.

Note: Such a set also satisfies Axiom P3' below, which follows from Axiom RW.

$\left(\mathrm{P} 3^{\prime}\right)$ For every height-two element $t$, the set $t^{\downarrow}$ is infinite.

Axiom RW is essential because it distinguishes $\operatorname{Spec}(\mathbb{Z}[x])$ from other similar prime spectra such as $\operatorname{Spec}(\mathbb{Q}[x, y])$; cf. [7]. The following theorem from R. Wiegand shows that the CZP axioms characterize $\operatorname{Spec}(\mathbb{Z}[x])$.

Theorem 2.5 ([7]). A poset $U$ satisfies the CZP axioms of Definition 2.4 if and only if $U$ is order isomorphic to $\operatorname{Spec}(\mathbb{Z}[x])$.

Remarks 2.6. The first two remarks are from $[4,5]$ :

(i) By Theorem 2.5 , every $h t(1,2)$-pair of $\operatorname{Spec}(\mathbb{Z}[x])$ has infinitely many radical elements in $\operatorname{Spec}(\mathbb{Z}[x])$.

(ii) Since $\operatorname{Spec}\left(\mathbb{Z}\left[\frac{1}{x}\right]\right) \cong \operatorname{Spec}(\mathbb{Z}[x])$, every ht(1,2)-pair of $\operatorname{Spec}\left(\mathbb{Z}\left[\frac{1}{x}\right]\right)$ has infinitely many radical elements in $\operatorname{Spec}\left(\mathbb{Z}\left[\frac{1}{x}\right]\right)$.

(iii) The following discussion shows how the existence of radical elements is important for showing that two posets $U$ and $V$ that both satisfy axioms for $\operatorname{Proj}(\mathbb{Z}[h, k])$ are order isomorphic. Since $\operatorname{Proj}(\mathbb{Z}[h, k])$ is a countable set, we would want to define an order-isomorphism $\varphi$ at each stage between finite subsets $F$ and $G$ of $U$ and $V$ respectively, and then extend $\varphi$ to $U$ and $V$. If $u_{0}$ and $v_{0}$ are the minimal elements of $U$ and $V$ respectively, $S$ is the set of height-one elements of $F, T$ is the set of height-two elements of $F$, and $\varphi$ is an orderisomorphism from $F=\{0\} \cup S \cup T$ in $U$ to $G=\{0\} \cup S^{\prime} \cup T^{\prime}$ in $V$, we would try to extend $\varphi$ so that a radical element for $(S, T)$ goes to a radical element for $\left(S^{\prime}, T^{\prime}\right)$. This is a simplification of the process; actually a height-one set $S$, and a height-two set $T$, might be enlarged first and $\varphi$ defined on enlarged ht(1,2)-pair before defining the map $\varphi$ on a radical element. The process is described more explicitly in Roger Wiegand's paper [6]. If we knew which pairs had radical elements, we could perhaps obtain such an order-isomorphism.

Notation 2.7. As mentioned in the introduction, the projective line over the integers, denoted by $\operatorname{Proj}(\mathbb{Z}[h, k])$, where $h$ and $k$ are indeterminates, has two standard interpretations as a partially ordered set. The first interpretation is from algebraic geometry; the second is more ring-theoretic and is used in this paper.

(i) $\operatorname{Proj}(\mathbb{Z}[h, k])$ is the set of all prime ideals of $\mathbb{Z}[h, k]$ generated by finite sets of homogeneous polynomials in the variables $h$ and $k$, but not those prime ideals containing both $h$ and $k$. 
(ii) $\operatorname{Proj}(\mathbb{Z}[h, k]):=\operatorname{Spec}(\mathbb{Z}[x]) \cup \operatorname{Spec}\left(\mathbb{Z}\left[\frac{1}{x}\right]\right)$, where $\operatorname{Spec}(\mathbb{Z}[x]) \cap \operatorname{Spec}\left(\mathbb{Z}\left[\frac{1}{x}\right]\right)$ is identified with $\operatorname{Spec}\left(\mathbb{Z}\left[x, \frac{1}{x}\right]\right)$. In this identification each prime ideal of the form $p \mathbb{Z}[x]$, where $p$ is a prime integer, is considered the same as $p \mathbb{Z}\left[\frac{1}{x}\right]$, and $f(x) \mathbb{Z}[x]$ is identified with $x^{-\operatorname{deg}(f)} f(x) \mathbb{Z}\left[\frac{1}{x}\right]$, for every irreducible polynomial $f(x)$ of $\mathbb{Z}[x] \backslash x \mathbb{Z}[x]$ with $\operatorname{deg}(f)>0$.

In particular, in the second view, if $f(x)=a_{n} x^{n}+\cdots+a_{1} x+a_{0} \in \mathbb{Z}[x]$ is irreducible, and $a_{n} \neq 0$ and $a_{0} \neq 0$, then we identify $(f(x)) \in \operatorname{Spec}(\mathbb{Z}[x])$ with $\left(\frac{1}{x^{n}} f(x)\right) \in \operatorname{Spec}\left(\mathbb{Z}\left[\frac{1}{x}\right]\right)$, written $(f(x)) \sim\left(\frac{1}{x^{n}} f(x)\right)$, where

$$
\frac{1}{x^{n}} f(x)=a_{0}\left(\frac{1}{x}\right)^{n}+\cdots+a_{n-1}\left(\frac{1}{x}\right)+a_{n} .
$$

Thus $\left(x^{2}+2 x+3\right) \mathbb{Z}[x] \sim\left(1+\frac{2}{x}+\frac{3}{x^{2}}\right) \mathbb{Z}\left[\frac{1}{x}\right]$. The only elements of $\operatorname{Spec}\left(\mathbb{Z}\left[\frac{1}{x}\right]\right)$ that are not in $\operatorname{Spec}(\mathbb{Z}[x])$ are the height-one prime $\frac{1}{x} \mathbb{Z}\left[\frac{1}{x}\right]$ and the height-two maximals $\left(p, \frac{1}{x}\right) \mathbb{Z}\left[\frac{1}{x}\right]$, where $p$ is a prime integer. Similarly $x \mathbb{Z}[x]$ is the only height-one element of $\operatorname{Spec}(\mathbb{Z}[x])$ not in $\operatorname{Spec}\left(\mathbb{Z}\left[\frac{1}{x}\right]\right)$, and $\{(p, x) \mathbb{Z}[x], p$ is a prime integer $\}$ is the set of all the height-two elements that are in $\operatorname{Spec}(\mathbb{Z}[x])$ but not in $\operatorname{Spec}\left(\mathbb{Z}\left[\frac{1}{x}\right]\right)$.

Here is an illustration of $\operatorname{Proj}(\mathbb{Z}[h, k])$ with this interpretation, from [5].

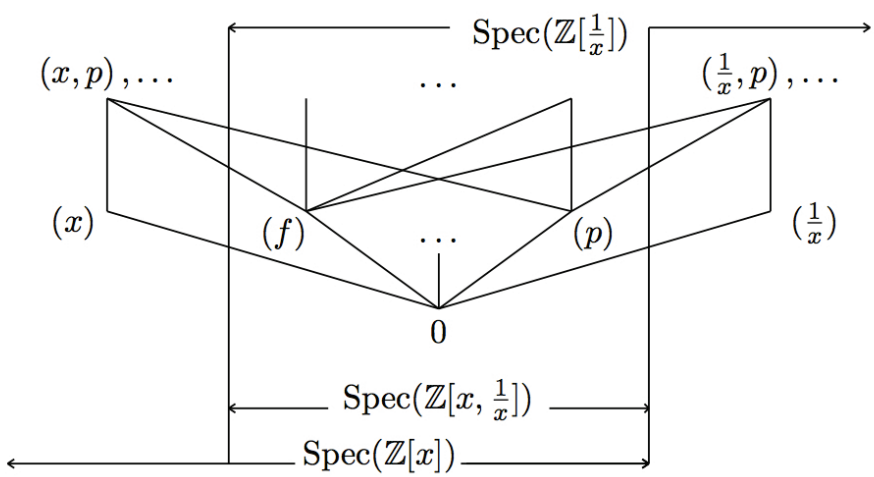

Figure 2. $\operatorname{Proj}(\mathbb{Z}[h, k])$.

The following proposition is useful for finding radical elements. The proof is straightforward and is omitted.

Proposition 2.8 ([1]). Let $f(x)=a_{n} x^{n}+\cdots+a_{0} \in \mathbb{Z}[x]$, where $a_{0}, \ldots, a_{n} \in \mathbb{Z}$ and $a_{n} \neq 0$, let $l(f)$ denote the leading coefficient $a_{n}$ of $f(x)$ and let $c(f)$ denote the constant term $a_{0}$ of $f(x)$.

(i) If $p$ is a prime integer, then

(a) $f(x) \in(x, p) \mathbb{Z}[x] \Leftrightarrow p \mid c(f)$;

(b) $(f(x))=\left(\frac{1}{x^{n}} f(x)\right) \subseteq\left(\frac{1}{x}, p\right) \mathbb{Z}\left[\frac{1}{x}\right] \Leftrightarrow p \mid l(f)$. 
(ii) If $f(x)$ is an irreducible element of $\mathbb{Z}[x]$ of positive degree in $x$, then

(a) $l(f)= \pm 1 \Leftrightarrow\left(f, \frac{1}{x}\right)^{\uparrow}=\emptyset \Leftrightarrow(f)^{\uparrow} \subseteq \operatorname{Spec}(\mathbb{Z}[x])$.

(b) $c(f)= \pm 1 \Leftrightarrow(f, x)^{\uparrow}=\emptyset \Leftrightarrow(f)^{\uparrow} \subseteq \operatorname{Spec}\left(\mathbb{Z}\left[\frac{1}{x}\right]\right)$.

By the following theorem, some adjustment of the CZP axioms of Definition 2.4 is necessary in order to describe $\operatorname{Proj}(\mathbb{Z}[h, k])$.

Theorem 2.9 ([5]). Proj( $\mathbb{Z}[h, k])$ satisfies Axioms P1-P4 of Definition 2.4, but does not satisfy Axiom RW of Definition 2.4. Thus $\operatorname{Proj}(\mathbb{Z}[h, k]) \neq \operatorname{Spec}(\mathbb{Z}[x])$.

The following example shows that the (RW) axiom fails for $\operatorname{Proj}(\mathbb{Z}[h, k])$ :

Example 2.10 ([5]). Let $S=\left\{\left(\frac{1}{x}\right),(2),(5)\right\}$ and $T=\left\{(x, 2),\left(\frac{1}{x}, 2\right),\left(\frac{1}{x}, 3\right)\right\}$ in $\operatorname{Proj}(\mathbb{Z}[h, k])$. Then the pair $(S, T)$ does not have a radical element in $\operatorname{Proj}(\mathbb{Z}[h, k])$.

Remark 2.11. If there is a radical element $w$ for a ht $(1,2)$-pair in $\operatorname{Proj}(\mathbb{Z}[h, k])$, then $w \notin S$. Otherwise, $w^{\uparrow} \subseteq T$ by Definition 2.2 (i), and this would imply $T$ is infinite by (P3) of Definition 2.4, a contradiction.

Our goal in this paper is to determine answers to Questions 2.12.

Questions 2.12. For which ht(1,2)-pairs of $\operatorname{Proj}(\mathbb{Z}[h, k])$ do radical elements exist? Which pairs have no radical element?

In what follows we obtain partial answers to these questions.

\section{The Coefficient Subset and Radical Elements of $\operatorname{Proj}(\mathbb{Z}[h, k])$}

In this section we give some more background and describe various ht $(1,2)$-pairs of $\operatorname{Proj}(\mathbb{Z}[h, k])$ in order to obtain partial answers to Question 2.12. In particular the "coefficient" subset $C_{0}$ of $\operatorname{Proj}(\mathbb{Z}[h, k])$ of prime ideals generated by prime elements of $\mathbb{Z}$ is relevant. It is more feasible that a ht $(1,2)$-pair $(S, T)$ has a radical element if, for every prime element $p$ of $\mathbb{Z}$ with $(p) \in S$, there is a maximal ideal $M \in T$ so that $p \in M$, cf. Proposition 3.5.

First in Proposition 3.1 we observe that some ht $(1,2)$-pairs $(S, T)$ inherit infinitely many radical elements in $\operatorname{Proj}(\mathbb{Z}[h, k])$ from $\operatorname{Spec}(\mathbb{Z}[x])$ or $\operatorname{Spec}\left(\mathbb{Z}\left[\frac{1}{x}\right]\right)$. This is because $\operatorname{Spec}(\mathbb{Z}[x])$ and $\operatorname{Spec}\left(\mathbb{Z}\left[\frac{1}{x}\right]\right)$ are CZP, cf. Theorem 2.5.

Proposition 3.1 ([5]). Every ht $(1,2)$-pair $(S, T)$ of $\operatorname{Proj}(\mathbb{Z}[h, k])$ has infinitely many radical elements in case (i) or (ii) hold:

(i) For every $s \in S$, $s^{\uparrow} \subseteq \operatorname{Spec}(\mathbb{Z}[x])$, and $T \subseteq \operatorname{Spec}(\mathbb{Z}[x])$.

(ii) For every $s \in S$, $s^{\uparrow} \subseteq \operatorname{Spec}\left(\mathbb{Z}\left[\frac{1}{x}\right]\right)$, and $T \subseteq \operatorname{Spec}\left(\mathbb{Z}\left[\frac{1}{x}\right]\right)$. 
Next we consider subsets of $\operatorname{Proj}(\mathbb{Z}[h, k])$ like the subsets of prime ideals generated by all prime integers of $\operatorname{Proj}(\mathbb{Z}[h, k])$. We consider the existence of radical elements for various ht $(1,2)$-pairs subject to conditions involving such a "coefficient" subset.

Definition 3.2 ([1]). Let $U$ be a poset of dimension two. A subset $C$ of height-one elements is called a coefficient subset of $U$ if

(i) For every $p \in C, p^{\uparrow}$ is infinite.

(ii) For every pair $p, q$ of distinct elements of $C, p \neq q \in C,(p, q)^{\uparrow}=\varnothing$.

(iii) $\bigcup_{p \in C} p^{\uparrow}=H_{2}(U)$.

(iv) For every $p \in C$ and $u \in H_{1}(U) \backslash C$, we have $(p, u)^{\uparrow} \neq \varnothing$, and $p^{\uparrow}=$ $\bigcup_{v \in H_{1}(U) \backslash C}(p, v)^{\uparrow}$.

Definition 3.3. Let $A \subseteq H_{1}(U)$, with $(a, b)^{\uparrow}=\emptyset$ for every $a, b \in A$. A coefficient subset $C$ is said to be attached to $A$ if, for every $p \in C$ and every $a \in A,\left|(p, a)^{\uparrow}\right|=1$.

Example 3.4. The set $C_{0}$ of all prime ideals of $\mathbb{Z}[x]$ generated by prime integers is a coefficient subset of $\operatorname{Proj}(\mathbb{Z}[h, k])$ attached to $\left\{(x),\left(\frac{1}{x}\right)\right\}$. It is also attached to $\left\{(x),\left(\frac{1}{x}\right),(x-1)\right\}$ or $\left\{(x),\left(\frac{1}{x}\right),(x+1)\right\}$.

Proposition 3.5 ([5]). Let $(S, T)$ be a ht(1,2)-pair and let $C$ be a coefficient subset of $\operatorname{Proj}(\mathbb{Z}[h, k])$. Suppose that there exist distinct elements $P_{0}$ and $P_{1}$ of $C$ such that $P_{0} \in S$ and $T \cap P_{0}^{\uparrow}=\emptyset$, but $T \cap P_{1}^{\uparrow} \neq \varnothing$. Then

(i) $(S, T)$ has no radical element except possibly $P_{1}$,

(ii) If $T \nsubseteq P_{1}^{\uparrow}$, then $P_{1}$ is not a radical element by Definition 2.2 (i),

(iii) There exists $Q \in H_{1}(\operatorname{Proj}(\mathbb{Z}[h, k])) \backslash C$ and $t \in P_{1}^{\uparrow} \cap Q^{\uparrow} \cap T$; thus $P_{1}$ is not a radical element for $(S \cup\{Q\}, T)$.

Proof. For (i), let $t \in T \cap P_{1}^{\uparrow}$. Suppose $Q$ were a radical element for $(S, T)$ and $Q \neq P_{1}$. If $Q \in C$, then $\left(Q, P_{1}\right)^{\uparrow}=\varnothing$ by (ii) of Definition 3.2, and so $t \notin Q^{\uparrow}$, a contradiction to Definition 2.2 for $Q$ a radical element. Thus $Q \notin C$, and so there exists $t^{\prime} \in\left(P_{0}, Q\right)^{\uparrow}$ by (4) of Definition 3.2. By hypothesis $t^{\prime} \in P_{0}^{\uparrow} \Longrightarrow t^{\prime} \notin T$, again contradicting that $Q$ is a radical element. Thus $(S, T)$ has no radical element except possibly $P_{1}$.

(ii) follows directly from Definition 2.2.

For (iv), since $P_{1}^{\uparrow}$ is infinite, there exists $t \in P_{1}^{\uparrow} \backslash T$. Now by (4) of Definition 3.2, there exists $Q \notin C$ with $t \in P_{1}^{\uparrow} \cap Q^{\uparrow}$. Thus $P_{1}$ is not a radical element for the pair $(S \cup\{Q\}, T)$.

Corollary 3.6 ([5]). Let $(S, T)$ be a ht(1,2)-pair in $\operatorname{Proj}(\mathbb{Z}[h, k])$. If $T \neq \emptyset$, then there exists a finite subset $S^{\prime}$ of $H_{1}(\operatorname{Proj}(\mathbb{Z}[h, k]))$ such that $S \subseteq S^{\prime}$ and $\left(S^{\prime}, T\right)$ has no radical element in $\operatorname{Proj}(\mathbb{Z}[h, k])$. 
The following results, Proposition 3.7 and Theorem 3.8, are used later to construct radical elements in various cases.

Proposition 3.7 ([2, page 102, Exercise 3]). Let $R$ be a domain and let $y$ be an indeterminate over $R$. Suppose (i) $\{a, b\}$ is an $R$-sequence or (ii) $(a, b)=R$, where $b \neq 0$. Then $(a+b y)$ is a prime ideal of $R[y]$.

Theorem 3.8 (Hilbert's Irreducibility Theorem, [3, page 141]). If $f \in \mathbb{Q}\left[x_{1}, \ldots, x_{r}, x\right]$ is an irreducible polynomial, then there exist $a_{1}, \ldots, a_{r} \in \mathbb{Q}$ such that $f\left(a_{1}, \ldots, a_{r}, x\right)$ remains irreducible in $\mathbb{Q}[x]$.

Meral Arnavut shows that the coefficient subset of $\operatorname{Proj}(\mathbb{Z}[h, k])$ is unique. She also gives partial results concerning the existence of radical elements in $\operatorname{Proj}(\mathbb{Z}[h, k])$.

Proposition 3.9 ([1]). $C_{0}:=\{p \mathbb{Z}[x] \mid p$ is a prime integer $\}$ is the only coefficient subset of $\operatorname{Proj}(\mathbb{Z}[h, k])$.

Proof. We sketch the proof from [1] briefly. If $\Gamma$ is a coefficient subset of $\operatorname{Proj}(\mathbb{Z}[h, k])$ such that $\Gamma \neq C_{0}$, then $\Gamma \cap C_{0}=\varnothing$. Let $p$ be a prime integer. Then $(p)^{\uparrow}$ is infinite and $\bigcup_{\gamma \in \Gamma}(\gamma, p)^{\uparrow}=(p)^{\uparrow}$. Hence $\Gamma$ is infinite. Therefore there exist distinct elements $\alpha$ and $\beta$ in $\Gamma \backslash C_{0} \backslash\left\{(x),\left(\frac{1}{x}\right)\right\}$; say $\alpha=(f(x)), \beta=(g(x))$, for two relatively prime irreducible polynomials $f(x)$ and $g(x)$ of $\mathbb{Z}[x]$ of positive degree. By Proposition 3.7, $(f+y g)$ is a prime ideal in $\mathbb{Z}[x, y]$, where $y$ is an indeterminate over $\mathbb{Z}[x]$. By Hilbert's Irreducibility Theorem 3.8, there exists a prime integer $p$ so that $f+p g$ is irreducible in $\mathbb{Z}[x]$. By Definition 3.2 (ii), no height-two prime ideals contain both $f$ and $g$. If $(f+p g) \notin \Gamma$, we contradict $(f, g)^{\uparrow}=\emptyset$. Hence $(f+p g) \in \Gamma$. But $(f, p)^{\uparrow} \subseteq(f)^{\uparrow} \cap(f+p g)^{\uparrow}=(f, f+p g)^{\uparrow}$. This contradicts Definition 3.2 (ii).

Remark 3.10 ([1]). Let $(S, T)$ be a ht(1,2)-pair in $\operatorname{Proj}(\mathbb{Z}[h, k])$. If $T \neq \emptyset$, then $(S, T)$ has at most one radical element in $C_{0}$.

Proposition 3.11 ([1]). Let $(S, T)$ be a ht $(1,2)$-pair in $\operatorname{Proj}(\mathbb{Z}[h, k])$. If $(S, T)$ has a radical element $Q$ in $\operatorname{Proj}(\mathbb{Z}[h, k])$ then either

(i) $S \cap C_{0} \subseteq \bigcup_{M \in T}\left(M^{\downarrow} \cap C_{0}\right)$ or

(ii) $Q \in C_{0}$.

In case (ii), if $T=\emptyset$, then $S \subseteq C_{0}$; if $T \neq \emptyset$, then $Q$ is the only radical element.

Sketch of the proof from [1]. If (i) fails, there exists $P \in S \cap C_{0}$ with $T \cap P^{\uparrow}=\emptyset$. Then $Q^{\uparrow} \cap P^{\uparrow}=\varnothing$. Thus, by Definition 3.2 (iv), $Q \in C_{0}$. Also $\bigcup_{s \in S}(s, Q)^{\uparrow} \subseteq$ $T \subseteq Q^{\uparrow}$. Thus if $T=\emptyset$, then $s \in C_{0}$, for all $s \in S$. If $T \neq \emptyset$, then $T$ contains an element of form $(f(x), p)$, where $p$ is a prime integer and either $f(x) \in \mathbb{Z}[x]$ has positive degree or $f(x)=\frac{1}{x}$. In either case $(f(x), p) \in Q^{\uparrow}$ implies $(p)=Q$, and so $Q$ is unique. 
Meral Arnavut notes that, if Condition 1 of Proposition 3.11 (i) is not satisfied, then it is difficult to find radical elements; cf. [1], and Proposition 3.5 and Proposition 3.11 of this paper.

Proposition 3.12 ([1]). Let $(S, T)$ be a $\mathrm{ht}(1,2)$-pair in $\operatorname{Proj}(\mathbb{Z}[h, k])$ such that

(i) $S \cap C_{0} \subseteq \bigcup_{M \in T}\left(M^{\downarrow} \cap C_{0}\right)$, and

(ii) $\bigcup_{s \in S}\left(s, \frac{1}{x}\right)^{\uparrow} \subseteq T$, or (ii') $\bigcup_{s \in S}(s, x)^{\uparrow} \subseteq T$.

Then $(S, T)$ has infinitely many radical elements in $\operatorname{Proj}(\mathbb{Z}[h, k])$.

Proof. We give the proof with hypothesis (ii); the proof for (ii') is similar (replace $\frac{1}{x}$ by $x$ ). Since $\left(s, \frac{1}{x}\right)^{\uparrow} \subseteq T$, for every $s \in S$, and $T$ is finite, $\left(\frac{1}{x}\right) \notin S$. Therefore $S \subseteq \operatorname{Spec}(\mathbb{Z}[x])$. If $T=\varnothing$, then, for every $s \in S, s^{\uparrow} \subseteq \operatorname{Spec}(\mathbb{Z}[x])$. Thus, by Proposition 3.1, $(S, T)$ has infinitely many radical elements in $\operatorname{Proj}(\mathbb{Z}[h, k])$ as desired. If $T \neq \varnothing$, let $p_{1}, \ldots, p_{r}$ denote the distinct positive prime integers such that

$$
\left\{\left(p_{1}\right), \ldots,\left(p_{r}\right)\right\}=\bigcup_{M \in T}\left(M^{\downarrow} \cap C_{0}\right) .
$$

Then, for each $t \in T$, some $p_{i} \in t$. Let $f_{1}, \ldots, f_{n}$ be irreducible polynomials of $\mathbb{Z}[x]$ of positive degree so that $S-C_{0}=\left\{\left(f_{1}\right), \ldots,\left(f_{n}\right)\right\}$. Let $T^{\prime}=T-\left(\frac{1}{x}\right)^{\uparrow}$. Therefore $T^{\prime} \subseteq \operatorname{Spec}(\mathbb{Z}[x])$ and $S \subseteq \operatorname{Spec}(\mathbb{Z}[x])$. Since $\operatorname{Spec}(\mathbb{Z}[x])$ is CZP, there are infinitely many radical elements for $\left(S, T^{\prime}\right)$ in $\operatorname{Spec}(\mathbb{Z}[x])$. By Proposition 3.10, $\left(S, T^{\prime}\right)$ has at most one radical element in $C_{0}$. Thus $\left(S, T^{\prime}\right)$ has infinitely many radical elements in $\operatorname{Spec}(\mathbb{Z}[x])-C_{0}$. Let $P_{0}$ be such a radical element; say $P_{0}=(f(x))$, where $f(x)$ is an irreducible polynomial of $\mathbb{Z}[x]$ of positive degree so that $f(x) \notin$ $x \mathbb{Z}[x] \cup f_{1} \mathbb{Z}[x] \cup \cdots \cup f_{n} \mathbb{Z}[x] \cup \mathbb{Z}$. Let $\lambda$ be a positive integer greater than the degree of $f(x)$. Then $f(x)$ and the product $p_{1} \cdots p_{r} f_{1} \cdots f_{n} x^{\lambda}$ are relatively prime in $\mathbb{Z}[x]$. By Proposition 3.7, $\left(y p_{1} \cdots p_{r} f_{1} \cdots f_{n} x^{\lambda}+f(x)\right)$ is a prime ideal of $\mathbb{Z}[x, y]$, where $y$ is an indeterminate over $\mathbb{Z}[x]$. By Hilbert's Irreducibility Theorem 3.8 , for each $\lambda$, there exists a prime integer $p_{\lambda}$ such that $g_{\lambda}(x)=p_{\lambda} p_{1} \cdots p_{r} f_{1} \cdots f_{n} x^{\lambda}+f(x)$ is an irreducible polynomial of $\mathbb{Z}[x]$; thus $w_{\lambda}:=\left(g_{\lambda}(x)\right)$ is a prime ideal of $\mathbb{Z}[x]$. For each $\lambda>\operatorname{deg}(f), w_{\lambda}$ is a radical element for $(S, T)$ in $\operatorname{Proj}(\mathbb{Z}[h, k])$. Thus $(S, T)$ has infinitely many radical elements.

\section{The Conjecture for $\operatorname{Proj}(\mathbb{Z}[h, k])$ and Previous Partial Results}

In Proposition 3.12 some conditions are given for a ht $(1,2)$-pair $(S, T)$ so that there are infinitely many radical elements. Item (ii) of Proposition 3.12 implies $\left(\frac{1}{x}\right) \notin S$ and item (ii)' implies that $(x) \notin S$. In either case, we get infinitely many radical elements in $\operatorname{Proj}(\mathbb{Z}[h, k])$. If both $(x)$ and $\left(\frac{1}{x}\right)$ belong to $S$, it is more difficult to find a radical 
element. The following conjecture first given by Aihua Li and Sylvia Wiegand, then adjusted by Meral Arnavut, addresses this case; cf. [1,5].

$\operatorname{Proj}(\mathbb{Z}[\boldsymbol{h}, \boldsymbol{k}])$ Conjecture 4.1 ([1]). Let $(S, T)$ be a ht $(1,2)$-pair in $\operatorname{Proj}(\mathbb{Z}[h, k])$. Assume

(i) $S \cap C_{0} \subseteq \bigcup_{m \in T}\left(m^{\downarrow} \cap C_{0}\right)$, and

(ii) $(x) \in S,\left(\frac{1}{x}\right) \in S$.

Then there exist infinitely many radical elements for $(S, T)$ in $\operatorname{Proj}(\mathbb{Z}[h, k])$.

It appears that some axiom regarding the existence of radical elements analogous to Axiom RW is necessary for $\operatorname{Proj}(\mathbb{Z}[h, k])$. The following axiom was proposed by $\mathrm{Li}$ and Wiegand and modified by Arnavut, cf. [1,5].

Axiom 4.2 (Axiom P5, [1]). Let $U$ be a poset of dimension two.

(P5a) There exist a unique coefficient subset $\Gamma$ of $U$ and special elements $u_{1}, u_{2} \in U$ such that $\left(u_{1}, u_{2}\right)^{\uparrow}=\varnothing$ and $\Gamma$ is attached to $u_{1}$ and $u_{2}$. (Thus, for every $\gamma \in \Gamma$, $\left|\left(\gamma, u_{1}\right)^{\uparrow}\right|=1=\left|\left(\gamma, u_{2}\right)^{\uparrow}\right|$.)

(P5b) Let $S$ be a nonempty finite subset of $H_{1}(U)$ and let $T$ be a nonempty finite subset of $\mathrm{H}_{2}(U)$.

(P5b.1) If $\gamma^{\uparrow} \cap T \neq \emptyset$, for every $\gamma \in S \cap \Gamma$, then there exist infinitely many radical elements for $(S, T)$.

(P5b.2) If there exists an element $\gamma \in S \cap \Gamma$ such that $\gamma^{\uparrow} \cap T=\emptyset$, then there is at most one possible radical element $\gamma_{0}$ for $(S, T)$, and $\gamma_{0} \in \Gamma \backslash S$.

Arnavut shows that Conjecture 4.1 implies Axiom P5 above for $\operatorname{Proj}(\mathbb{Z}[h, k])$ and that $U:=\operatorname{Proj}(\mathbb{Z}[h, k]) \backslash C_{0}$ is CZP; cf. [1]. We believe that this will lead to a complete characterization of $\operatorname{Proj}(\mathbb{Z}[h, k])$.

We give a special case of the Conjecture 4.1 when $T=\emptyset$.

Proposition 4.3 ([1]). Suppose $S$ is a finite subset of $H_{1}(\operatorname{Proj}(\mathbb{Z}[h, k]))$ of the form

$$
S=\left\{(x),\left(\frac{1}{x}\right),\left(f_{1}\right), \ldots,\left(f_{n}\right)\right\},
$$

where $f_{1}, \ldots, f_{n}$ are monic irreducible polynomials of $\mathbb{Z}[x]$ of positive degree. Then $(S, \varnothing)$ has infinitely many radical elements in $\operatorname{Proj}(\mathbb{Z}[h, k])$.

Remark 4.4. Similarly one can find infinitely many radical elements if $T=\emptyset$ and $S$ is a finite subset of $H_{1}(\operatorname{Proj}(\mathbb{Z}[h, k]))$ such that $S=\left\{(x),\left(\frac{1}{x}\right),\left(f_{1}\right), \ldots,\left(f_{n}\right)\right\}$, where $f_{1}, \ldots, f_{n}$ are irreducible polynomials in $\mathbb{Z}[x]$ of positive degree with $c\left(f_{i}\right)= \pm 1$. However we do not know what happens when $T=\emptyset, c\left(f_{i}\right) \neq \pm 1$ and $\ell\left(f_{i}\right) \neq \pm 1$. In this case if there is a radical element $(g(x))$ where $g(x)$ is an irreducible polynomial, 
then $c(g)= \pm 1, \ell(g)= \pm 1$ and $\left(g(x), f_{i}(x)\right)=1$. If we could find such radical elements, the conjecture would hold for $T=\emptyset$. This might help prove the conjecture for the $T \neq \varnothing$ case as well.

Meral Arnavut introduces the following notation and gives some partial results related to the conjecture, recorded here as Theorem 4.6, cf. [1].

Notation 4.5. Let $T$ be a nonempty finite subset of $H_{2}(\operatorname{Proj}(\mathbb{Z}[h, k]))$.

Let $F:=\{p \in \mathbb{Z}, p$ prime $\mid(x, p) \in T\}$ and let $G:=\left\{p \in \mathbb{Z}, p\right.$ prime $\mid\left(\frac{1}{x}, p\right) \in$ $T\}$. Then $A_{1}:=F \backslash G, A_{2}:=F \cap G$, and $A_{3}:=G \backslash F$ are disjoint sets.

Define $a_{i}:=\prod_{p \in A_{i}} p$, for $i=1,2,3$. Thus $a_{1}, a_{2}$ and $a_{3}$ are pairwise relatively prime integers. For each $i$, if $A_{i}=\varnothing$, we set $a_{i}=1$. Now let $n \in \mathbb{N}$, and define $f_{n}(x) \in \mathbb{Z}[x]$ by

$$
f_{n}(x):= \begin{cases}a_{3} x^{n}+a_{1}, & \text { if } F \cap G=\varnothing\left(\text { i.e., } a_{2}=1\right), \\ a_{2}^{n} a_{3} x^{2}+a_{1} a_{3} x+a_{2}^{n} a_{1}, & \text { if } F \cap G \neq \varnothing .\end{cases}
$$

Theorem 4.6 ([1]). Let $(S, T)$ be an ht(1,2)-pair in $\operatorname{Proj}(\mathbb{Z}[h, k])$ and let $F, G, A_{1}$, $A_{2}, A_{3}, a_{1}, a_{2}, a_{3}$ and $f_{n}$ be as in as in Notation 4.5. Suppose

- $T \subseteq(x)^{\uparrow} \cup\left(\frac{1}{x}\right)^{\uparrow}$,

- $S \cap C_{0} \subseteq\{(p) \mid p \in F \cup G\}$,

- $(x) \in S,\left(\frac{1}{x}\right) \in S$.

Then:

(i) If $\left(s, f_{n}\right)^{\uparrow} \subseteq T$, for every $s \in S \backslash\left(C_{0} \cup\left\{(x),\left(\frac{1}{x}\right)\right\}\right)$, then $\left(f_{n}\right)$ is a radical element for $(S, T)$ in $\operatorname{Proj}(\mathbb{Z}[h, k])$.

(ii) If $S \backslash C_{0}=\left\{(x),\left(\frac{1}{x}\right)\right\}$, then $(S, T)$ has infinitely many radical elements in $\operatorname{Proj}(\mathbb{Z}[h, k])$.

(iii) If $F \cap G=\emptyset$ and, for every irreducible polynomial $f(x)$ of $\mathbb{Z}[x]$ such that $(f) \in S \backslash\left(C_{0} \cup\left\{(x),\left(\frac{1}{x}\right)\right\}\right)$, (a) or (b) holds, that is,

(a) $\ell(f)$ is a unit, and $a_{1}$ divides every coefficient of $f(x)$ except $\ell(f)$,

(b) $c(f)$ is a unit, and $a_{3}$ divides every coefficient of $f(x)$ except $c(f)$,

then $(S, T)$ has infinitely many radical elements in $\operatorname{Proj}(\mathbb{Z}[h, k])$.

(iv) If $F \cap G=\emptyset$ and $S \backslash C_{0}=\left\{(x),\left(\frac{1}{x}\right)\right\} \cup\{(x+\alpha)\}$, for some $\alpha \in \mathbb{Z}$ such that $a_{1}$ and $\alpha$ are relatively prime, then $(S, T)$ has a radical element in $\operatorname{Proj}(\mathbb{Z}[h, k])$.

Corollary 4.7 ([1]). Let

$$
\begin{aligned}
S & =\left\{\left(p_{1}\right), \ldots,\left(p_{n}\right),(x),\left(\frac{1}{x}\right),\left(f_{1}\right), \ldots,\left(f_{m}\right)\right\}, \\
T & =\left\{\left(x, p_{1}\right), \ldots,\left(x, p_{l}\right),\left(\frac{1}{x}, p_{l+1}\right), \ldots,\left(\frac{1}{x}, p_{n}\right)\right\},
\end{aligned}
$$

where $0 \leq l \leq n, p_{1}, \ldots, p_{n}$ are distinct prime integers. 
(i) If $f_{i}(x) \in \mathbb{Z}[x]$ has the form $x^{d_{i}}+p_{1} \cdots p_{l} b_{i}$, for some $d_{i} \in \mathbb{N}$ and $b_{i} \in \mathbb{Z}$ with $1 \leq i \leq m$, then $(S, T)$ has infinitely many radical elements.

(ii) If $f_{i}(x) \in \mathbb{Z}[x]$ has the form $b_{i} p_{l+1} \cdots p_{n} x^{d_{i}}+1$, for some $d_{i} \in \mathbb{N}$ and $b_{i} \in \mathbb{Z}$ with $1 \leq i \leq m$, then $(S, T)$ has infinitely many radical elements.

\section{New Results Supporting the Conjecture}

In this section we give some new results that further support Conjecture 4.1. We consider various different types of $h t(1,2)$-pairs in $\operatorname{Proj}(\mathbb{Z}[h, k])$.

Theorem 5.1. Let $(S, T)$ be an $\mathrm{ht}(1,2)$-pair in $\operatorname{Proj}(\mathbb{Z}[h, k])$. Suppose

(i) $T \subseteq(x)^{\uparrow}$,

(ii) $S \cap C_{0} \subseteq\{(p) \mid(x, p) \in T, p \in \operatorname{Spec}(\mathbb{Z})\}$,

(iii) $S \backslash C_{0}=\left\{(x),\left(\frac{1}{x}\right)\right\} \cup\left\{\left(a_{1} x+1\right), \ldots,\left(a_{m} x+1\right)\right\}$ for some $a_{i} \in \mathbb{Z}, i=1, \ldots, m$.

Then $(S, T)$ has infinitely many radical elements in $\operatorname{Proj}(\mathbb{Z}[h, k])$.

Proof. Assume that $T \neq \emptyset$. Since $T \subseteq(x)^{\uparrow}$ by (i), we have $F \neq \emptyset$ where $F:=\{p \in$ $\mathbb{Z}, p$ prime $\mid(x, p) \in T\}$. Let $\lambda \in \mathbb{N}$ be such that $\lambda \geq 2$. Define

$$
g_{\lambda}(x):=x^{m+\lambda}+b\left(a_{1} x+1\right) \cdots\left(a_{m} x+1\right) \in \mathbb{Z}[x]
$$

where the $a_{i}$ are as in (iii) and $b=\prod_{p \in F} p$. We show $w_{\lambda}=\left(g_{\lambda}(x)\right)$ is a radical element for $(S, T)$ in $\operatorname{Proj}(\mathbb{Z}[h, k])$. By Eisenstein's Criteria, $g_{\lambda}(x)$ is irreducible in $\mathbb{Z}[x]$. To see that $w_{\lambda}$ satisfies Definition 2.2, let $t \in T$. Then $t=(x, p)$, for some $p \in F$. But $p \mid c\left(g_{\lambda}\right)$, and so $w_{\lambda} \subset t$, for every $t \in T$. Let $s \in S$ and let $M \in H_{2}(\operatorname{Proj}(\mathbb{Z}[h, k]))$ be such that $g_{\lambda}(x) \in M$ and $s \subset M$. We consider $\left(w_{\lambda}, s\right)^{\uparrow}$ for all possible types of $s \in S$ :

(i) Since $\left(g_{\lambda}(x), a_{i} x+1\right)=(1),\left(g_{\lambda}(x), a_{i} x+1\right)^{\uparrow}=\emptyset$, for all $i=1, \ldots, m$.

(ii) Since $\left(g_{\lambda}(x), \frac{1}{x}\right)=(1),\left(\frac{1}{x}, g_{\lambda}(x)\right)^{\uparrow}=\emptyset$.

(iii) Since $\left(g_{\lambda}(x), x\right)=(x, b), M=(x, p)$, for some $p \in F$, and hence $M \in T$.

(iv) Since $\left(g_{\lambda}(x), p\right)=\left(x^{m+\lambda}, p\right)$, for $p \in F$ such that $p \mid b, M=(x, p)$.

Thus $M \in T$, and so $\left(p, g_{\lambda}(x)\right)^{\uparrow} \in T$.

Thus $w_{\lambda}=\left(g_{\lambda}(x)\right)$ is a radical element for $(S, T)$ in $\operatorname{Proj}(\mathbb{Z}[h, k])$ for each $\lambda \in \mathbb{N}$, and so there are infinitely many radical elements $w_{\lambda}$ for $(S, T)$.

If $T=\emptyset$, then take $b=1$ and define

$$
g_{\lambda}(x):=x^{m+\lambda}+\left(a_{1} x+1\right) \cdots\left(a_{m} x+1\right) \in \mathbb{Z}[x] .
$$

Similarly $w_{\lambda}=\left(g_{\lambda}(x)\right)$ is a radical element for $(S, T)$ in $\operatorname{Proj}(\mathbb{Z}[h, k])$, for $\lambda \in \mathbb{N}$. 
Remark 5.2. Similarly there exist infinitely many radical elements for a ht(1,2)-pair $(S, T)$ in $\operatorname{Proj}(\mathbb{Z}[h, k])$ satisfying the following:

(i) $T \subseteq\left(\frac{1}{x}\right)^{\uparrow}$,

(ii) $S \cap C_{0} \subseteq\left\{(p) \mid\left(\frac{1}{x}, p\right) \in T, p \in \operatorname{Spec}(\mathbb{Z})\right\}$,

(iii) $S \backslash C_{0}=\left\{(x),\left(\frac{1}{x}\right)\right\} \cup\left\{\left(x+a_{1}\right), \ldots,\left(x+a_{m}\right)\right\}$ for some $a_{i} \in \mathbb{Z}, i=1, \ldots, m$.

Proposition 5.3. Consider

$$
\begin{aligned}
& S=\left\{\left(p_{1}\right), \ldots,\left(p_{n}\right),(x),\left(\frac{1}{x}\right),\left(x+a_{1}\right), \ldots,\left(x+a_{m}\right)\right\} \\
& T=\left\{\left(x, p_{1}\right),\left(\frac{1}{x}, p_{2}\right), \ldots,\left(\frac{1}{x}, p_{n}\right),\left(x+a_{1}, p_{1}\right), \ldots,\left(x+a_{m}, p_{1}\right)\right\}
\end{aligned}
$$

where $p_{1}, \ldots, p_{n}$ are distinct prime integers, $n>1, a_{1}, \ldots, a_{m} \in \mathbb{Z}$ and $\left(a_{k}, p_{1}\right)=$ 1 for each $k=1, \ldots, m$. Then $(S, T)$ has infinitely many radical elements in $\operatorname{Proj}(\mathbb{Z}[h, k])$.

Proof. Let $\lambda \in \mathbb{N}$. Define $h_{\lambda}(x)=b^{\lambda} x^{\lambda}\left(x+a_{1}\right) \ldots\left(x+a_{m}\right)+p_{1}^{\lambda}$ where $b=$ $\prod_{i=2}^{n} p_{i}$. We show that $w_{\lambda}=\left(h_{\lambda}(x)\right)$ is a radical element for $(S, T)$ in $\operatorname{Proj}(\mathbb{Z}[h, k])$. First, by Eisenstein's Criteria for $\mathbb{Z}\left[\frac{1}{x}\right], h_{\lambda}(x)$ is irreducible in $\mathbb{Z}\left[\frac{1}{x}\right]$. Also $w_{\lambda} \subset t$ for all $t \in T$. Let $s \in S$ and $M \in H_{2}(\operatorname{Proj}(\mathbb{Z}[h, k]))$ be such that $h_{\lambda}(x) \in M$ and $s \subset M$. We consider $\left(w_{\lambda}, s\right)^{\uparrow}$ for all possible types of $s \in S$ :

Since $\left(h_{\lambda}(x), x+a_{k}\right)=\left(x+a_{k}, p_{1}^{\lambda}\right), M=\left(x+a_{k}, p_{1}\right)$ is the only maximal ideal that contains $\left(h_{\lambda}(x), x+\alpha_{k}\right)$, for $k=1, \ldots, m$, that is, $\left(h_{\lambda}(x), x+a_{k}\right)^{\uparrow} \in T$.

Since $\left(h_{\lambda}(x), x\right) \subseteq\left(x, p_{1}^{\lambda}\right), M=\left(x, p_{1}\right)$ is the only maximal ideal that contains $\left(h_{\lambda}(x), x\right)$, that is, $\left(h_{\lambda}(x), x\right)^{\uparrow} \in T$.

Since $\left(h_{\lambda}(x), \frac{1}{x}\right)=\left(b^{\lambda}, \frac{1}{x}\right)$, for $i=2, \ldots, n, M$ has form $\left(\frac{1}{x}, p_{i}\right)$ for some $i$, and the $\left(\frac{1}{x}, p_{i}\right)$ are the only maximal ideals that contain $\left(h_{\lambda}(x), \frac{1}{x}\right)$, for $i=2, \ldots, n$.

Since $\left(h_{\lambda}(x), p_{1}\right)=\left(b^{\lambda} x^{\lambda}\left(x+a_{1}\right) \ldots\left(x+a_{m}\right)\right), M=\left(x, p_{1}\right)$ or $M=(x+$ $\left.a_{k}, p_{1}\right) \in T$, for some $k=1, \ldots, m$, and these are the only maximal ideals that contain $\left(h_{\lambda(x)}, p_{1}\right)$.

If $s=\left(p_{i}\right)$, for $i=2, \ldots, n$, then we get $\left(h_{\lambda}(x), p_{i}\right)=\left(p_{1}^{\lambda}, p_{i}\right)=(1)$ since $p_{i} \mid b$. Therefore, for each $\lambda \in \mathbb{N}, w_{\lambda}$ is a radical element for $(S, T)$.

Proposition 5.4. Let $\alpha_{1}, \ldots, \alpha_{m} \in \mathbb{Z}$ be such that $\operatorname{gcd}\left(\alpha_{1}, \ldots, \alpha_{m}\right) \neq 1$. Suppose that $p_{1}, \ldots, p_{k}$ are all the prime integers that are factors of any of the $\alpha_{i}$ and that $p_{1}$ divides each of the $\alpha_{i}$. Say each $\alpha_{i}=p_{1}^{e_{i 1}} \cdots p_{k}^{e_{i k}}$, for some $e_{i \ell} \geq 0$. Then let $n \geq 1$ and choose prime integers $q_{1}, \ldots, q_{n}$ distinct from $p_{1}, \ldots, p_{k}$. Let

$$
\begin{aligned}
& B_{1}:=\left\{\left(x, q_{j}\right),\left(x+\alpha_{i}, q_{j}\right)\right\}_{\substack{1 \leq i \leq m \\
1 \leq j \leq n}},
\end{aligned}
$$

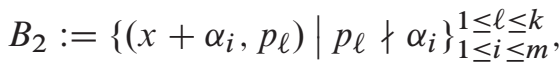

$$
\begin{aligned}
& B_{3}:=\left\{\left(x, p_{1}\right) \ldots\left(x, p_{k}\right)\right\} \text {, }
\end{aligned}
$$


and set

$$
\begin{aligned}
S & =\left\{(x),\left(\frac{1}{x}\right),\left(x+\alpha_{1}\right) \ldots,\left(x+\alpha_{m}\right)\right\} \cup\left\{\left(q_{j}\right)\right\}_{1 \leq j \leq n} \cup\left\{\left(p_{\ell}\right)\right\}_{1 \leq \ell \leq k}, \\
T & =B_{1} \cup B_{2} \cup B_{3} .
\end{aligned}
$$

Then $(S, T)$ has infinitely many radical elements in $\operatorname{Proj}(\mathbb{Z}[h, k])$.

Proof. Let $\lambda \in \mathbb{N}$. Define $a_{0}:=\prod_{\left(\begin{array}{c}1 \leq \ell \leq k \\ 1 \leq j \leq n\end{array}\right)} p_{\ell} \cdot q_{j}$ and $h_{\lambda}(x)=x^{\lambda}\left(x+\alpha_{1}\right) \cdots(x+$ $\left.\alpha_{m}\right)+a_{0}$.

We show that $w_{\lambda}=\left(h_{\lambda}(x)\right)$ is a radical element for $(S, T)$ in $\operatorname{Proj}(\mathbb{Z}[h, k])$.

Note that $h_{\lambda}(x)$ is irreducible by Eisenstein in $\mathbb{Z}[x]$, since $\operatorname{gcd}\left(\alpha_{1}, \ldots, \alpha_{m}\right) \neq 1$ and $p_{1} \mid \operatorname{gcd}\left(\alpha_{1}, \ldots, \alpha_{m}\right)$. Also $w_{\lambda} \subset t$ for all $t \in T$.

Let $s \in S$ and $t \in H_{2}(\operatorname{Proj}(\mathbb{Z}[h, k]))$ be such that $h_{\lambda}(x) \in t$ and $s \subseteq t$.

If $s=\left(q_{j}\right),\left(h_{\lambda}, q_{j}\right)=\left(x^{\lambda}\left(x+\alpha_{1}\right) \cdots\left(x+\alpha_{m}\right), q_{j}\right)$, for $j=1, \ldots, n$, and so $\left(h_{\lambda}, q_{j}\right)^{\uparrow}=\left\{\left(x, q_{j}\right),\left(x+\alpha_{1}, q_{j}\right), \ldots,\left(x+\alpha_{m}, q_{j}\right)\right\} \subset B_{1}$. Similarly, if $s=\left(p_{\ell}\right)$, then $\left(h_{\lambda}, p_{\ell}\right)^{\uparrow} \subset B_{2} \cup B_{3}$, for $\ell=1, \ldots, k$. If $s=(x)$, then $\left(h_{\lambda}, x\right) \subset\left(x, q_{j}\right)$, for all $j, 1 \leq j \leq n$ and also $\left(h_{\lambda}, x\right) \subset\left(x, p_{\ell}\right), 1 \leq \ell \leq k$. If $s=\left(\frac{1}{x}\right)$, then $\left(h_{\lambda}, \frac{1}{x}\right)=(1)$ because $h_{\lambda}$ is a monic polynomial of $\mathbb{Z}[x]$. If $s=\left(x+\alpha_{i}\right)$ for some $i, 1 \leq i \leq m$, then $\left(h_{\lambda}, x+\alpha_{i}\right) \subset\left(x+\alpha_{i}, q_{j}\right) \in B_{1}$ for some $j, 1 \leq j \leq \ell$. If $p_{\ell} \nmid \alpha_{i}$, then $\left(h_{\lambda}, x+\alpha_{i}\right) \subset\left(x+\alpha_{i}, p_{\ell}\right) \in B_{2}$ and if $p_{\ell} \mid \alpha_{i},\left(h_{\lambda}, x+\alpha_{i}\right) \subset\left(x+\alpha_{i}, p_{\ell}\right) \in B_{3}$, for each $i, 1 \leq i \leq m$ and $1 \leq \ell \leq k$.

Therefore, in any of the latter cases, $\left(h_{\lambda}, x+\alpha_{i}\right)^{\uparrow} \subset T$, for $i, 1 \leq i \leq m$. Thus $w_{\lambda}$ is a radical element for $(S, T)$ in $\operatorname{Proj}(\mathbb{Z}[h, k])$. Now, since $\lambda \in \mathbb{N}$, there are infinitely many $w_{\lambda}$ in $\operatorname{Proj}(\mathbb{Z}[h, k])$ and so $(S, T)$ has infinitely many radical elements.

Theorem 5.5. There exist infinitely many radical elements for every $\mathrm{ht}(1,2)$-pair in $\operatorname{Proj}(\mathbb{Z}[h, k])$ of the form $S=\left\{(x),\left(\frac{1}{x}\right),\left(p_{1}\right), \ldots,\left(p_{n}\right)\right\}$ and $T=\left\{\left(x, p_{1}\right), \ldots\right.$, $\left.\left(x, p_{n}\right),\left(\frac{1}{x}, p_{1}\right), \ldots,\left(\frac{1}{x}, p_{n}\right)\right\}$, where $p_{1}, \ldots, p_{n}$ are distinct prime integers.

Proof. First consider the subsets

$$
\begin{array}{rlrl}
S_{x} & :=\left\{(x),\left(p_{1}\right), \ldots,\left(p_{n}\right)\right\}, & T_{x}:=\left\{\left(x, p_{1}\right), \ldots,\left(x, p_{n}\right)\right\}, \\
S_{\frac{1}{x}}:=\left\{\left(\frac{1}{x}\right),\left(p_{1}\right), \ldots,\left(p_{n}\right)\right\}, & T_{\frac{1}{x}}:=\left\{\left(\frac{1}{x}, p_{1}\right), \ldots,\left(\frac{1}{x}, p_{n}\right)\right\} .
\end{array}
$$

Then $S_{x} \cup T_{x} \subseteq \operatorname{Spec}(\mathbb{Z}[x])$. Thus we see that for every $\lambda \in \mathbb{N}$,

$$
f_{\lambda}(x):=x^{\lambda}+p_{1} \cdots p_{n} \in \mathbb{Z}[x]
$$

$\left(f_{\lambda}\right)$ is a radical element for $\left(S_{x}, T_{x}\right)$ in $\operatorname{Spec}(\mathbb{Z}[x])$ since $f(x)$ is irreducible by Eisenstein. Similarly for

$$
h_{\lambda}\left(\frac{1}{x}\right):=p_{1} \cdots p_{n}+\frac{1}{x^{\lambda}} \in \mathbb{Z}\left[\frac{1}{x}\right]
$$


$\left(h\left(\frac{1}{x}\right)\right)$ is a radical element for $\left\{S_{\frac{1}{x}}, T_{\frac{1}{x}}\right\}$. We identify $h\left(\frac{1}{x}\right)$ with

$$
g_{\lambda}(x):=x^{\lambda} h_{\lambda}\left(\frac{1}{x}\right)=x^{\lambda} p_{1} \cdots p_{n}+1 \in \mathbb{Z}[x] .
$$

Let $y$ be another indeterminate over $\mathbb{Z}[x]$ and let $k(x)=p_{1} \cdots p_{n} x^{\lambda}$. Then

$$
f_{\lambda}(x) g_{\lambda}(x)=x^{2 \lambda} p_{1} \cdots p_{n}+\left(\left(p_{1} \cdots p_{n}\right)^{2}+1\right) x^{\lambda}+p_{1} \cdots p_{n} .
$$

Since $f_{\lambda}(x) g_{\lambda}(x)$ and $k(x)$ are relatively prime elements of $\mathbb{Z}[x], f_{\lambda}(x) g_{\lambda}(x)+$ $y k(x)$ is a prime ideal in $\mathbb{Z}[x, y]$ by Proposition 3.7. Thus there exists a prime integer $q$ so that $f_{\lambda}(x) g_{\lambda}(x)+q\left(p_{1} \cdots p_{n} x^{\lambda}\right)$ is irreducible in $\mathbb{Z}[x]$ by Hilbert's Irreducibility Theorem 3.8.

We show that $w_{\lambda}:=\left(r_{\lambda}(x)\right)=\left(f_{\lambda}(x) g_{\lambda}(x)+q k(x)\right)$ is a radical element for $(S, T)$ for all $\lambda \in \mathbb{N}$. First observe

$$
r_{\lambda}(x)=p_{1} \cdots p_{n} x^{2 \lambda}+\left(\left(p_{1} \cdots p_{n}\right)^{2}+1+q p_{1} \cdots p_{n}\right) x^{\lambda}+p_{1} \cdots p_{n} .
$$

It is easy to see that $w_{\lambda} \subset t$ for every $t \in T$, since $p_{i} \mid \ell(r)$ and $p_{i} \mid c(r)$, for $i=1, \ldots, n$. Also $\left(r_{\lambda}, x\right) \subseteq\left(p_{i}, x\right)$ for all $i=1, \ldots, n$. Similarly $\left(r_{\lambda}, \frac{1}{x}\right) \subseteq$ $\left(\frac{1}{x}, p_{i}\right)$, for all $i=1, \ldots, n$. Moreover $\left(r_{\lambda}, p_{i}\right)=\left(x^{\lambda}, p_{i}\right)$ and so $\left(x, p_{i}\right)$ is the only maximal element that contains $\left(r_{\lambda}, p_{i}\right)^{\uparrow}$, for $i=1, \ldots, n$. Thus $\left(r_{\lambda}, p_{i}\right)^{\uparrow} \in T$, for $i=1, \ldots, n$. Therefore $w_{\lambda}$ is a radical element for each $\lambda \in \mathbb{N}$.

Example 5.6. There are infinitely many radical elements for every ht(1,2)-pair in $\operatorname{Proj}(\mathbb{Z}[h, k])$ of the form

$$
\begin{aligned}
S & =\left\{(x),\left(\frac{1}{x}\right),(2),(3),(5)\right\}, \\
T & =\left\{(x, 2),(x, 3),(x, 5),\left(\frac{1}{x}, 2\right),\left(\frac{1}{x}, 3\right),\left(\frac{1}{x}, 5\right)\right\} .
\end{aligned}
$$

First consider the following subsets as in the previous proof of Theorem 5.5:

$$
\begin{aligned}
S_{x} & =\{(x),(2),(3),(5)\}, & T_{x} & =\{(x, 2),(x, 3),(x, 5)\}, \\
S_{\frac{1}{x}} & =\left\{\left(\frac{1}{x}\right),(2),(3),(5)\right\}, & T_{\frac{1}{x}} & =\left\{\left(\frac{1}{x}, 2\right),\left(\frac{1}{x}, 3\right),\left(\frac{1}{x}, 5\right)\right\} .
\end{aligned}
$$

Then $S_{x} \cup T_{x} \subseteq \operatorname{Spec} \mathbb{Z}[x]$ and for every $\lambda \in \mathbb{N}, f_{\lambda}(x):=x^{\lambda}+30$ in $\mathbb{Z}[x]$ generates a radical element for $\left(S_{x}, T_{x}\right)$ in $\operatorname{Spec}\left(\mathbb{Z}\left[\frac{1}{x}\right]\right)$. Similarly $h_{\lambda}\left(\frac{1}{x}\right):=30+$ $\frac{1}{x^{\lambda}} \in \mathbb{Z}\left[\frac{1}{x}\right]$ is a radical element for $\left(S_{\frac{1}{x}}, T_{\frac{1}{x}}\right)$. We identify $h_{\lambda}\left(\frac{1}{x}\right)$ with $g_{\lambda}(x):=$ $30 x^{\lambda}+1 \in \mathbb{Z}[x]$. Let $y$ be another indeterminate over $\mathbb{Z}[x]$. Since $f_{\lambda}(x) g_{\lambda}(x)$ and $30 x^{\lambda}$ are relatively prime elements of $\mathbb{Z}[x],\left(f_{\lambda}(x) g_{\lambda}(x)+y\left(30 x^{\lambda}\right)\right)$ is a prime ideal of $\mathbb{Z}[x, y]$ by Proposition 3.7. There exists a prime integer $q$ so that $f_{\lambda}(x) g_{\lambda}(x)+$ $q\left(30 x^{\lambda}\right)$ is irreducible in $\mathbb{Z}[x]$ by Hilbert's Irreducibility Theorem 3.8. Therefore $w=$ $\left(f_{\lambda}(x) g_{\lambda}(x)+30 q x^{\lambda}\right)$ is a radical element for each $\lambda \in \mathbb{N}$. 
In [1], Arnavut raises some questions about particular ht $(1,2)$-pairs in $\operatorname{Proj}(\mathbb{Z}[h, k])$. We consider one such unanswered question below.

Question 5.7. Does $(S, T)$ have a radical element if

$$
\begin{aligned}
S & =\left\{\left(p_{1}\right), \ldots,\left(p_{n}\right),(x),\left(\frac{1}{x}\right),(x-a),(x-b)\right\}, \\
T & =\left\{\left(x, p_{1}\right), \ldots,\left(x, p_{\ell}\right),\left(\frac{1}{x}, p_{\ell+1}\right), \ldots,\left(\frac{1}{x}, p_{n}\right)\right\},
\end{aligned}
$$

where $0 \leq \ell \leq n, \operatorname{gcd}\left(a b, p_{1} \cdots p_{\ell}\right)=1$, and the $p_{i}$ are distinct prime integers for $i=1, \ldots, n$ ?

Theorem 5.8 answers Question 5.7 in a special case.

Theorem 5.8. Assume $a$ and $b$ are relatively prime integers and let $S$ and $T$ be the following subsets of $\operatorname{Proj}(\mathbb{Z}[h, k])$ :

$$
\begin{aligned}
S & :=\left\{\left(p_{1}\right), \ldots,\left(p_{n}\right),(x),\left(\frac{1}{x}\right),(x-a),(x-b)\right\}, \\
T & :=\left\{\left(x, p_{1}\right), \ldots,\left(x, p_{\ell}\right),\left(\frac{1}{x}, p_{\ell+1}\right), \ldots,\left(\frac{1}{x}, p_{n}\right)\right\},
\end{aligned}
$$

where $0 \leq \ell \leq n, \operatorname{gcd}\left(a b, p_{1} \ldots p_{\ell}\right)=1$, and the $p_{i}$ are distinct prime integers for $i=1, \ldots, n$. Suppose also that $\mathbf{p q}$ divides $\left(1-\mathbf{p}^{t}\right)\left(b^{2}+a b+a^{2}\right)+\mathbf{q} a^{3} b^{3}$ and $(1-$ $\left.\mathbf{p}^{t}+\mathbf{q} b^{2} a^{2}\right)(b+a)$ where $\mathbf{p}=p_{1} \ldots p_{\ell}, \mathbf{q}=p_{\ell+1} \ldots p_{n}, t=\operatorname{lcm}\left(\phi\left(a^{2}\right), \phi\left(b^{2}\right)\right)$, and $\phi$ is the Euler phi function. Then $(S, T)$ has infinitely many radical elements in $\operatorname{Proj}(\mathbb{Z}[h, k])$.

Proof. Consider the polynomial $g(x ; u, v, w)$ of the form

$$
g(x ; u, v, w)=\mathbf{q} x^{4}+(\mathbf{p q} u) x^{3}+(\mathbf{p q} v) x^{2}+(\mathbf{p q} w) x+(\mathbf{p})^{t}
$$

where $t=\operatorname{lcm}\left(\phi\left(a^{2}\right), \phi\left(b^{2}\right)\right)$.

We show there exist infinitely many triples $u, v$, and $w \in \mathbb{Z}$ such that $(g(x ; u, v, w))$ is a radical element for $(S, T)$ in $\operatorname{Proj}(\mathbb{Z}[h, k])$.

First, by Euler's theorem, $(\mathbf{p})^{\phi\left(a^{2}\right)} \equiv 1\left(\bmod a^{2}\right)$ and $(\mathbf{p})^{\phi\left(b^{2}\right)} \equiv 1\left(\bmod b^{2}\right)$, since $\operatorname{gcd}(a, \mathbf{p})=1$ and $\operatorname{gcd}(b, \mathbf{p})=1$. Thus $\mathbf{p}^{t}-1 \equiv 0\left(\bmod a^{2} b^{2}\right)$, that is, $a^{2} b^{2}$ divides $\mathbf{p}^{t}-1$.

To find $u, v$, and $w \in \mathbb{Z}$, we solve the system of linear equations $g(a ; u, v, w)=1$ and $g(b ; u, v, w)=1$; that is,

$$
\begin{aligned}
& \mathbf{q} a^{4}+\mathbf{p q} a^{3} u+\mathbf{p q} a^{2} v+\mathbf{p q} a w+\mathbf{p}^{t}=1, \quad \text { and } \\
& \mathbf{q} b^{4}+\mathbf{p q} b^{3} u+\mathbf{p q} b^{2} v+\mathbf{p q} b w+\mathbf{p}^{t}=1 .
\end{aligned}
$$


This becomes:

$$
\begin{aligned}
& u+\frac{v}{a}+\frac{w}{a^{2}}=\frac{1-\mathbf{p}^{t}-\mathbf{q} a^{4}}{\mathbf{p q} a^{3}} \\
& u+\frac{v}{b}+\frac{w}{b^{2}}=\frac{1-\mathbf{p}^{t}-\mathbf{q} b^{4}}{\mathbf{p q} b^{3}}
\end{aligned}
$$

By subtracting (5.2) from (5.1), we get

$$
v\left(\frac{b-a}{a b}\right)+w\left(\frac{b^{2}-a^{2}}{a^{2} b^{2}}\right)=\frac{\left(1-p^{t}\right)\left(b^{3}-a^{3}\right)+q(b-a) a^{3} b^{3}}{p q a^{3} b^{3}} .
$$

After simplifying (5.3), we deduce

$$
v+w\left(\frac{a+b}{a b}\right)=\frac{\left(1-p^{t}\right)\left(b^{2}+a b+a^{2}\right)+q a^{3} b^{3}}{p q a^{2} b^{2}} .
$$

Therefore, for every $w=a b k$ where $k \in \mathbb{Z}$, we get

$$
v=\frac{\left(1-p^{t}\right)\left(b^{2}+a b+a^{2}\right)+q a^{3} b^{3}}{p q a^{2} b^{2}}-(a+b) k .
$$

Similarly, by eliminating $v$, and letting $w=a b k$ for $k \in \mathbb{Z}$, we get

$$
u=-\frac{\left(1-\mathbf{p}^{t}+\mathbf{q} b^{2} a^{2}\right)(b+a)}{\mathbf{p q} a^{2} b^{2}}-k .
$$

Note that pq divides $\left(1-\mathbf{p}^{t}\right)\left(b^{2}+a b+a^{2}\right)+\mathbf{q} a^{3} b^{3}$ and $\left(1-\mathbf{p}^{t}+\mathbf{q} b^{2} a^{2}\right)(b+a)$. Moreover $a^{2} b^{2}$ divides $1-p^{t}$. Hence $u$ and $v$ are integers in (5.5) and (5.6).

Now we claim that for every triple of integers $u, v$ and $w$ that we have found above, the polynomial $g(x ; u, v, w):=\mathbf{q} x^{4}+(\mathbf{p q} u) x^{3}+(\mathbf{p q} v) x^{2}+(\mathbf{p q} w) x+(\mathbf{p})^{t} \in \mathbb{Z}[x]$ generates a radical element for $(S, T)$. First $g(x ; u, v, w)$ is irreducible by Eisenstein's Criteria in $\mathbb{Z}\left[\frac{1}{x}\right]$. Since $c(g)=\mathbf{p}^{t}$ and $\ell(g)=\mathbf{q}$, we have $(g(x ; u, v, w)) \subseteq z, \forall$ $z \in T$. Consider $(g(x ; u, v, w), s)^{\uparrow}$ for each $s \in S$ :

For $s=(x),(g(x ; u, v, w), x)=\left(\mathbf{p}^{t}, x\right) \subseteq\left(p_{i}, x\right) \in T$, where $i=1, \ldots, \ell$.

For $s=\left(\frac{1}{x}\right)$, we have $\left(g(x ; u, v, w), \frac{1}{x}\right)=\left(\mathbf{q}, \frac{1}{x}\right)$. The only maximal ideals containing $\left(\mathbf{q}, \frac{1}{x}\right)$ are $\left(p_{j}, \frac{1}{x}\right) \in T$, for $j=\ell+1, \ldots, n$.

For $s=\left(p_{i}\right)$, where $i=1, \ldots, \ell$, we get $\left(g(x ; u, v, w), p_{i}\right)=\left(\mathbf{q} x^{4}, p_{i}\right)$. The only maximal ideals containing $\left(\mathbf{q} x^{4}, p_{i}\right)$ are $\left(x, p_{i}\right)$, since $\left(\mathbf{q}, p_{i}\right)=(1)$.

For $s=\left(p_{j}\right)$, where $j=\ell+1, \ldots, n$, we have $\left(g(x ; u, v, w), p_{j}\right)=\left(\mathbf{p}^{t}, p_{j}\right)=(1)$.

For $s=(x-a)$, we have $(g(x ; u, v, w), x-a)=1$ since $g(a ; u, v, w)=(1)$.

Similarly, for $s=(x-b)$, we get $(g(x ; u, v, w), x-b)=(1)$ since $g(b ; u, v, w)=1$.

Therefore we conclude that $(g(x ; u, v, w))$ is a radical element for $(S, T)$, for all $u, v$ and $w \in \mathbb{Z}$ as chosen in the proof. Thus there are infinitely many radical elements for this $(S, T)$-pair in $\operatorname{Proj}(\mathbb{Z}[h, k])$. 
Example 5.9. For $S=\left\{(2),(3),(x),\left(\frac{1}{x}\right),(x-5),(x-7)\right\}$ and $T=\left\{(x, 2),\left(\frac{1}{x}, 3\right)\right\}$, the polynomial $g(x ; u, v, w):=3 x^{4}+6 u x^{3}+6 v x^{2}+6 w x+2^{420} \in \mathbb{Z}[x]$ generates a radical element for $(S, T)$ for $w=0$,

$$
\begin{aligned}
& u=\frac{\left(1-2^{420}+3 \cdot 5^{2} \cdot 7^{2}\right)(5+7)}{2 \cdot 3 \cdot 5^{2} \cdot 7^{2}} \in \mathbb{Z}, \quad \text { and } \\
& v=\frac{\left(1-2^{420}\right)\left(5^{2}+35+7^{2}\right)+3 \cdot 5^{3} \cdot 7^{3}}{2 \cdot 3 \cdot 5^{2} \cdot 7^{2}} \in \mathbb{Z} .
\end{aligned}
$$

Note that $u$ and $v$ are integers since $5^{2} \cdot 7^{2}$ divides $1-2^{420}$, and also $2 \cdot 3=6$ divides the numerators $\left(1-2^{420}+3 \cdot 5^{2} \cdot 7^{2}\right)(5+7)$ and $\left(1-2^{420}\right)\left(5^{2}+35+7^{2}\right)+3 \cdot 5^{3} \cdot 7^{3}$.

Also, if $w=5 \cdot 7 \cdot k=35 k$, for $k \in \mathbb{Z}$, then we get different integers $u$ and $v$, that is, $g(x ; u, v, w)$ generates a different radical element for every $k \in \mathbb{Z}$. Therefore $(S, T)$ has infinitely many radical elements in $\operatorname{Proj}(\mathbb{Z}[h, k])$.

\section{Summary and Questions}

As the reader can see, there is still much to be done for the characterization of $\operatorname{Proj}(\mathbb{Z}[h, k])$. In particular, the determination of which $(S, T)$-pairs have radical elements appears to be very challenging. In the future we hope to address some of the following questions:

Questions 6.1. (i) In the setting of Theorem 4.7 with

$$
\begin{aligned}
S & =\left\{\left(p_{1}\right), \ldots,\left(p_{n}\right),(x),\left(\frac{1}{x}\right),\left(f_{1}\right), \ldots,\left(f_{m}\right)\right\}, \\
T & =\left\{\left(x, p_{1}\right), \ldots,\left(x, p_{l}\right),\left(\frac{1}{x}, p_{l+1}\right), \ldots,\left(\frac{1}{x}, p_{n}\right)\right\},
\end{aligned}
$$

where $0 \leq l \leq n, p_{1}, \ldots, p_{n}$ are distinct prime integers, is there a radical element for $(S, T)$ if

(a) The leading coefficient of $f_{1} \cdots f_{m}$ is not a unit and $p_{l+1} \cdots p_{n}$ does not divide the leading coefficient of $f_{i}$, for some $i$ ?

(b) The constant coefficient of $f_{1} \cdots f_{m}$ is not a unit and $p_{1} \cdots p_{l}$ does not divide the constant coefficient of $f_{i}$, for some $i$ ?

(c) $\operatorname{gcd}\left(p_{1} \cdots p_{n}, \ell\left(f_{1} \cdots f_{m}\right)\right)=1$ and $\operatorname{gcd}\left(p_{1} \cdots p_{n}, c\left(f_{1} \cdots f_{m}\right)\right)=1$ ?

(ii) Does the $(S, T)$-pair in Theorem 5.8 have a radical element if we remove some assumptions?

(iii) Let $u_{1}, \ldots, u_{n}, v_{1}, \ldots, v_{m} \in H_{1}(\operatorname{Proj}(\mathbb{Z}[h, k]))-C_{0}$, and let $P \in C_{0}$. Does there exist a $Q \in C_{0}$ such that $\left|\bigcup_{i=1}^{n}\left(u_{i}, P\right)^{\uparrow}\right|=\left|\bigcup_{j=1}^{m}\left(v_{j}, P\right)^{\uparrow}\right|$ ?

(iv) What happens if we change $T$ ? 
Acknowledgments. We are grateful to Sylvia Wiegand for her help with this article, which is part of Celikbas's thesis. We wish to thank Jim Coykendall for helping us to find an example for Question 5.7 in Section 5. We also would like to thank the referee whose comments and suggestions have greatly improved the presentation of the paper.

\section{Bibliography}

[1] Arnavut, M., The projective line over the integers. Arab J Sci Eng Sect C Theme Issues. 2001;26:1,31-44,1319-8025.

[2] Kaplansky, I., Commutative rings, Boston: Allyn and Bacon; 1970.

[3] Lang, S., Diophantine geometry. New York: Interscience; 1959.

[4] Li, A., Wiegand. S., Polynomial behavior of prime ideals in polynomial rings and the projective line over $\mathbb{Z}$. Proceedings, Factorization in Integral Domains; 1996, Iowa City, IA. Lecture notes in Pure and applied mathematics. New York: Marcel Dekker; 1997. p. 383-399.

[5] Li, A., Wiegand, S., Prime ideals in birational extensions of polynomials over the integers. J Pure Appl Algebra 1998;130:313-324.

[6] Wiegand, R., Homomorphisms of affine surfaces over a finite field. J Lond Math Soc (2). $1978 ; 18: 28-32$.

[7] Wiegand, R., The prime spectrum of a two-dimensional affine domain. J. Pure Appl Algebra. 1986;40:209-214.

\section{Author Information}

Ela Celikbas, Department of Mathematics, University of Nebraska-Lincoln, Lincoln, NE, USA.

E-mail: s-ecelikb1@math.unl.edu

Christina Eubanks-Turner, Department of Mathematics, University of Louisiana at Lafayette, Lafayette, LA, USA.

E-mail: ceturner@louisiana.edu 
